\title{
Interseccionalidad en las desigualdades sociales para la realización de actividad física en Argentina
}

\author{
Intersectionality of Social Inequalities in \\ Physical Activity in Argentina
}

Interseccionalidade nas desigualdades sociais para a realização de atividade física na Argentina

Matías Salvador Ballesteros, PhD $^{1 *}$

Betina Freidin, Lic. PhD 1

Agustín Wilner, Lic ${ }^{2}$

Lucas Fernández Rendina²

Recibido: 18 de febrero de 2019 - Aceptado: $1^{\circ}$ de octubre de 2019

Doi: https://doi.org/10.12804/revistas.urosario.edu.co/revsalud/a.8777

Para citar este artículo: Ballesteros MS, Freidin B, Wilner A, Fernández L. Interseccionalidad en las desigualdades sociales para la realización de actividad física en Argentina. Rev. Cienc. Salud. 2020; 18 (1): 134-151. https://doi.org/10.12804/revistas.urosario.edu.co/ revsalud/a.8777

\section{Resumen}

Introducción: el objetivo del artículo es analizar las desigualdades en cuanto a la realización de actividad física para mejorar la condición física o para hacer deporte, determinadas por distintos factores sociales, geográficos y demográficos, así como por algunas de las interacciones entre estos factores. Para ello, se recurrió a la estrategia de análisis cuantitativo de datos secundarios. La fuente utilizada es la Encuesta Nacional de Factores de Riesgo, realizada sobre una muestra probabilística de hogares urbanos de Argentina, en el 2009. Desarrollo: para examinar los datos, se realizó un análisis descriptivo bivariado y, luego, regresiones logísticas binarias, en las que se incorporaron interacciones, para poner a prueba diversas hipótesis sobre el efecto de las distintas variables independientes, así como de su interseccionalidad. Conclusiones: los resultados muestran la importancia de considerar un enfoque interseccional e incorporar interacciones en las regresiones logísticas binarias para comprender la naturaleza multiplicativa de las desigualdades en la práctica de actividad física, que los modelos multivariados aditivos no pueden captar.

Palabras clave: actividad física; ejercicio; inequidad social; interseccionalidad; Argentina.

1 Carrera de Sociología e Instituto de Investigaciones Gino Germani (IIGG), Universidad de Buenos Aires. Consejo Nacional de Investigaciones Científicas y Técnicas (CONICET).

Autor de correspondencia: matiballesteros@yahoo.com.ar

2 Carrera de Sociología e Instituto de Investigaciones Gino Germani (IIGG), Universidad de Buenos Aires. 
Introduction: The aim of this article is to analyze inequalities in the practice of physical activity for enhancing physical conditions or for doing sports, generated by different social, geographical and demographical determinants, as well as some of their interactions. Development: A quantitative analysis of secondary data was conducted. The source is the National Survey of Risk Factors, based on a probabilistic sample of urban households in Argentina, in 2009. Firstly, it is presented a descriptive bivariate analysis and, secondly, binary logistic regressions to test hypotheses on the net effect of different independent variables are shown and incorporated interactions within them to observe their intersectionality. Conclusions: Findings show the importance of considering an intersectional approach to account for the multiplicative nature of inequalities in the practice of physical activity, which most used multivariate models fail to show.

Keywords: Physical activity; exercise; social inequity; intersectionality; Argentina.

\section{Resumo}

Introdução: o objetivo do artigo é analisar as desigualdades na realização de atividade física para melhorar a condição física ou para fazer esporte, geradas por distintos determinantes sociais, geográficos e demográficos, assim como algumas de suas interações. Para isto se recorreu à estratégia de análise quantitativo de dados secundários. A fonte utilizada é a Enquete Nacional de Fatores de Risco, realizada sobre uma amostra probabilística de lares urbanos da Argentina no ano 2009. Desenvolvimento: para examinar os dados se realizou uma análise descritiva bivariada e logo regressões logísticas binarias, nas que se incorporaram interações em seu interior, para pôr a prova diversas hipóteses sobre o efeito das distintas variáveis independentes assim como de sua interseccionalidade. Conclusões: os resultados mostram a importância de considerar um enfoque interseccional e incorporar interações ao interior das regressões logísticas binárias para compreender a natureza multiplicativa das desigualdades na prática de atividade física, que os modelos multivariados aditivos não podem captar.

Palavras-chave: atividade física; exercício; inequidade social; interseccionalidade; Argentina.

\section{Introducción}

Existen desigualdades sociales que determinan las posibilidades de desarrollar estilos de vida Esaludables en el espacio urbano, incluyendo el ejercicio físico y el deporte. Desde la epidemiología crítica, la salud colectiva, la salud de la población (population health) y la sociología médica, se ha puesto el foco del análisis en los aspectos colectivos; es decir, en los modos de vida de la población, estructurados por las relaciones de clase, género, etarias y étnicas. Los modos afectan las posibilidades individuales y familiares de desarrollar hábitos protectores de la salud $(1,2)$. Así mismo, los estudios han centrado su atención en aspectos territoriales que condicionan el acceso diferencial a la infraestructura y a las políticas públicas que promueven la realización de actividades recreativas y deportivas; y en la oferta privada, que se localiza preferencialmente en los barrios y áreas de clase media y alta (3). Otros aspectos ambientales físicos y construidos del territorio también limitan las posibilidades de realizar actividad física en espacios públicos, entre ellos la violencia interpersonal y la exposición al riesgo ambiental (3-6). En este sentido, los grupos marginalizados sufren 
una doble desventaja para cuidar su salud: la marginación individual, por su desventaja estructural, y la inequidad derivada de su localización territorial (7). Así, es posible distinguir entre factores "objetivos" y "subjetivos", que afectan diferencialmente la realización de actividad física (8). Entre los primeros se encuentra la accesibilidad a las instalaciones para hacer ejercicio, o las oportunidades que un espacio brinda para realizarlo; y entre los segundos, se encuentran aspectos estéticos de los espacios y si éstos son percibidos como seguros (8). A su vez, quienes perciben apoyo de políticas públicas para la actividad física y el deporte y ventajas en su entorno personal tienen mayores probabilidades de realizar actividad física (9).

Un conjunto abundante de estudios cuantitativos internacionales analiza cómo los distintos determinantes sociales (clase social, nivel educativo, ingreso, género, etnia), demográficos y geográficos generan desigualdades en las posibilidades de realizar actividad física recreacional (10-12). Estos trabajos observan el efecto neto de cada una de las variables independientes, una vez controlado el efecto de las restantes. A su vez, trabajos recientes ponen de relieve la interseccionalidad de los determinantes sociales en salud, entendiendo que estos son interdependientes; además, enfatizan en cómo la combinación de distintas posiciones sociales generadas por estructuras macrosociales corresponde con experiencias diferenciales a nivel microsocial, al reforzar distintos ejes de privilegio u opresión (13-15). Por ejemplo, si bien han disminuido las desigualdades de género en la realización de deporte en la clase media, continúan siendo muy intensas en la clase trabajadora, entre las minorías étnicas y si las mujeres tienen hijos pequeños $(12,14-16)$. Ello se debe a que las mujeres con mayor desventaja estructural cuentan con menos tiempo libre por ldoble jornada(trabajo productivo y reproductivo) o por el poco dinero con el que cuentan para destinar al ocio (14-17). De este modo, considerar a las mujeres como grupo homogéneo puede conducir a errores, puesto que las desigualdades de clase acentúan las de género en relación con la práctica deportiva y el ocio (16). Entre los recursos de clase se encuentran la capacidad de disponer de dinero para comprar el tiempo de mujeres de clase trabajadora como ayuda en el hogar y el cuidado de los hijos, tener más recursos materiales para el ocio y disponer de transporte privado $(16,18)$.

Otros estudios han analizado la relación entre el género, la edad y la actividad deportiva en las sociedades europeas en un contexto demográfico marcado por el envejecimiento poblacional y por los efectos socioculturales de la sportification - la difusión masiva de la oferta de actividades deportivas y recreativas asociadas con el tiempo libre y la diversificación de ámbitos para practicarlas (12). En Alemania, considerando la población de cincuenta años y más, los resultados en torno al género no son concluyentes, ya que el único patrón común hallado en diversos estudios es la menor actividad deportiva en las mujeres con respecto a los hombres en el grupo poblacional correspondiente a personas con ochentas años o más, lo que se explica por un efecto de cohorte, en la medida en que las mujeres de edades más avanzadas fueron socializadas en una cultura del deporte altamente masculinizada (12). A su vez, entre los adultos mayores se observa una correlación positiva entre la clase social, 
el nivel educativo y la actividad deportiva, relación que se acentúa para las mujeres (12); es decir, los efectos de la estratificación social son más importantes para las mujeres que para los hombres. Con relación al género y la edad, en España, a la inversa de la población general, las mujeres de sesenta y cinco años y más realizan más actividad física que los hombres (19).

En la literatura se han propuesto dos hipótesis contrapuestas para explicar la interacción entre edad, género y ejercicio físico: por un lado, se establece que las diferencias de género observadas en estudios transversales podrían acortarse en el caso de los grupos más jóvenes, y esta hipótesis plantea el efecto de cohorte, pues considera que las mujeres de edades más avanzadas fueron socializadas en una cultura deportiva masculinizada (12); por otro lado, se hipotetiza que las desigualdades de género tienden a disminuir entre los grupos etarios de mayor edad (19), lo que se debería a distintos factores confluyentes relativos al ciclo vital. Por un lado, sobre las mujeres recaen mayores cargas relacionadas con el cuidado de los niños menores, y las mayores desigualdades de género entre los grupos más jóvenes estarían relacionadas con la falta de tiempo de las mujeres con hijos. Como la carga se reduce a medida que los hijos son mayores, en los grupos etarios de edad más avanzada las diferencias disminuirían. Por otro lado, se plantea que la asociación de la masculinidad hegemónica con el deporte no solo limita la participación de las mujeres, sino también la de los adultos mayores en general, en tanto han "ido perdiendo los atributos de fuerza, resistencia, fortaleza, etc.” (20). Las diferencias de género en la realización de deporte serían más fuertes entre los grupos jóvenes. En esta línea de ideas, cabe señalar además que, a medida que aumenta la edad, es más frecuente que se dejen de realizar determinados deportes asociados con lo masculino debido a impedimentos físicos asociados a una mayor carga de morbilidad (21), lo que podría conllevar, además, una menor desigualdad de género en edades más avanzadas. Si bien estos antecedentes dan cuenta de la importancia del abordaje interseccional de los determinantes sociales, pocos trabajos cuantitativos lo han incorporado en modelos de análisis multivariado (13).

En Argentina, se analizaron los determinantes sociales que generan desigualdades en la práctica de ejercicio físico (22-26). Según datos de una encuesta nacional realizada en el año 2017 entre población urbana, el $80 \%$ de los individuos pertenecientes a sectores con nivel socioeconómico más bajos reportaron no haber realizado ningún ejercicio físico en la última semana, frente al $46 \%$ correspondiente a la población de niveles socioeconómicos más altos. Así mismo, se encontró que el $69 \%$ de las mujeres no realizaron actividad física en la semana previa a la encuesta, frente al $60 \%$ de los varones; y el $81 \%$ de los residentes en áreas más precarias no realizaron actividad física, frente al $52 \%$ de los que disponen de mejores condiciones de residencia (25) ${ }^{3}$. Sin embargo, poco se ha avanzado en los relevamientos nacionales en el análisis empírico con modelos multivariados que incorporen la interseccionalidad de los determinantes sociales.

3 Se consideró ejercicio a la actividad física que mejora y mantiene la aptitud física, la salud y el bienestar del individuo. 
Tomando como base la Encuesta Nacional de Factores de Riesgo (ENFR) del año 2009, en este artículo analizamos el impacto de los distintos determinantes sociales, geográficos y demográficos para la práctica de ejercicio físico, así como algunas de sus interacciones. En particular, analizamos si las desigualdades de género se mantienen constantes entre distintas posiciones sociales y grupos etarios, o si estas se intensifican para algunos de ellos. Para ello ponemos a pruebas las siguientes hipótesis:

- Hipótesis 1: Los factores socioeconómicos, geográficos y demográficos inciden en las posibilidades de realizar actividad física (AF) para mejorar la condición física o hacer deporte (мсг/D). Cada factor tiene su peso propio una vez controlado el efecto de los restantes.

- Hipótesis 2: Las diferencias de género en las posibilidades de realizar AF para MCF/D son más importantes entre los sectores de menos recursos.

Con respecto a la interacción entre género y edad para la realización de AF para McF/D, ponemos a prueba dos hipótesis contrapuestas:

- Hipótesis 3a: Las diferencias de género tienden a disminuir para los grupos más jóvenes.

- Hipótesis 3b: Las diferencias de género tienden a disminuir para los grupos etarios de mayor edad.

\section{Metodología}

Tos datos analizados provienen de la segunda Encuesta Nacional de Factores de Riesgo

L(ENFR) realizada en Argentina de forma conjunta por el Instituto Nacional de Estadísticas y Censos (INDEC) y el Ministerio de Salud, en el año 2009. El relevamiento utilizó una muestra probabilística multietápica (por conglomerados y estratificado). Se encuestaron 34732 personas de dieciocho años y más, residentes en viviendas particulares de localidades urbanas de 5000 habitantes y más (27). Dado que se trató de un muestreo no proporcional, los realizadores de la encuesta desarrollaron factores de ponderación. En nuestro análisis, utilizamos los factores de ponderación sin expandir los resultados a toda la población.

El cuestionario individual de la ENFR 2009 contiene un primer bloque de preguntas que permiten clasificar a la población adulta según su nivel de actividad física durante la semana anterior al relevamiento. Allí se pregunta por toda la actividad física (AF) realizada sin diferenciar el tipo. Luego se incluye otro bloque de preguntas sobre la actividad física realizada durante el último mes y por los distintos tipos, diferenciando entre actividad física doméstica, laboral, por traslado/desplazamiento, y para mejorar la condición física/hacer deporte (мсF/D). El análisis que presentamos en este artículo se basa en esta última pregunta, ${ }^{4}$ pues

4 En un trabajo previo realizamos un análisis descriptivo de las otras preguntas mencionadas anteriormente (28). 
se considera que es la variable dependiente en los distintos modelos multivariados. Ello se debe a que, si bien la actividad física es todo movimiento corporal que involucra músculos esqueléticos y requiere de gasto energético, algunas funciones son obligatorias (por ejemplo, las ocupacionales o las tareas del hogar), mientras otras, como el deporte, son de carácter voluntario, generan disfrute y, por lo tanto, pueden contribuir a un mayor bienestar emocional y social (29). Estas últimas actividades se realizan en el tiempo libre y dependen de las motivaciones y de recursos individuales, comunitarios y residenciales $(5,7,30){ }^{5}$

En nuestro análisis comenzamos examinando la relación bivariada entre la realización de AF para MCF/D y un conjunto de variables independientes como sexo, grupo etario, nivel educativo, quintil de ingreso per cápita del hogar, hogar con necesidades básicas insatisfechas (NBI) y región de residencia. Luego realizamos regresiones logísticas binarias paso por paso que incorporan las variables anteriores por bloque. A partir de la regresión logística podemos saber cuál es el efecto de una variable independiente, una vez controlada el resto. En un primer modelo introducimos las distintas variables socioeconómicas y la región de residencia, mientras que en un segundo modelo, además, incluimos al sexo y el grupo etario. A partir de estos dos primeros modelos podremos poner a prueba la hipótesis 1.

Estos modelos muestran las desigualdades de forma aditiva; es decir, indican el efecto adicional de cada una de las variables una vez controlado el efecto del resto (conocido como efectos principales) (13). Sin embargo, los modelos no nos indican si se produce un efecto diferencial al considerar la combinación de distintas posiciones sociales (si existen efectos multiplicativos). Por ejemplo, no nos permiten analizar si las diferencias de género se mantienen constantes o aumentan según el quintil de ingreso del hogar, el nivel educativo o el grupo etario del entrevistado. Para ello, incorporamos el análisis de las interacciones en los modelos 3, 4 y 5, que nos permitirán poner a prueba las hipótesis 2 y 3. En una regresión logística binaria la interacción entre dos variables independientes es significativa cuando el efecto de una de ellas sobre la dependiente estudiada varía según los valores de la otra variable independiente. En este caso, la nueva variable incluida pasa a ser "un modificador" o "moderador” del efecto que la primera variable independiente ejercía sobre la dependiente (31).

Al incluir en una regresión logística binaria interacciones entre dos variables cualitativas, surgen uno o más coeficientes (según la cantidad de categorías de las variables) que indican el ratio que resulta al dividir dos chances relativas (odds ratio) (32). Por ejemplo, en el modelo 3 (tabla 2) que presentamos más adelante, realizamos una interacción entre las variables sexo y quintil de ingreso per cápita del hogar. La variable moderadora introducida es el quintil

5 Cabe señalar que la forma de analizar la AF para MCF/D tiene una serie de limitaciones. Ello se debe a que no estamos analizando el tipo de actividad, la intensidad, la cantidad de días que se realiza, ni la motivación para realizarla. De esta manera, los datos que examinados en este artículo no distinguen entre quienes, por ejemplo, realizaron una caminata durante el último mes y quienes practican periódicamente deportes. 
de ingreso, y tomamos como categoría de referencia al primer quintil, mientras que mujer es la categoría de referencia para el sexo . La expresión que el modelo elabora al comparar las diferencias de probabilidad que hombres y mujeres tienen dentro de los quintiles 5 y 1 por ejemplo, tendrá la siguiente forma:

$$
\begin{aligned}
\operatorname{Exp}(B)=\frac{1}{2}= & \frac{\frac{\text { Odds Hombres para A.F }}{\text { Odds Mujeres para A.F } . F^{\text {Duntil }} 5}}{\frac{\text { Odds Hombres para A.F }}{\text { Dentrodel quintill } 1}} \\
= & 0,54 \text { (ver Tabla 2, modelo } 3)
\end{aligned}
$$

En el numerador se encuentran las chances relativas de realizar actividad física para hombres y mujeres, pero solo para aquellos individuos que integran el quintil 5, mientras que en el denominador se encuentran las del quintil 1. Si en una "ratio de odds ratios" - como la presentada - el resultado es igual a 1, o el intervalo de confianza elegido incluye el 1, podríamos decir que las diferencias de género entre el quinto y el primer quintil no serían significativas (31). Esto nos informaría que el efecto del género sobre una persona a la hora de realizar AF para MCF/D no se vería modificado por pertenecer a un quintil de bajos ingresos o al de más altos ingresos. Por el contrario, si no pasa por el 1, son significativas. En nuestro ejemplo, el resultado (0.54) es menor a 1, lo que indica que las chances a favor de los hombres frente a las mujeres para realizar actividad física son mayores en el primer quintil (or2) que en el quinto quintil (or1).

Finalmente, cabe señalar que también se modifica la interpretación de los coeficientes de las variables que son parte de la interacción (por ejemplo, para la lectura de la variable "sexo" o de los diferentes quintiles de ingreso en el modelo 3). Los coeficientes de una de las variables de la interacción representan el odds ratio original que se leía en los modelos sin interacciones, pero solo para la categoría de referencia de la otra variable que es parte de la interacción (31). A modo de ejemplo, en nuestro modelo 3 interactúan las variables sexo y quintil de ingreso. Cuando leamos las chances relativas de la variable sexo, fuera de los términos de la interacción, estaremos comparando las chances relativas para practicar actividad física entre hombres y mujeres, pero solo dentro del quintil 1 (categoría de referencia para la variable quintil de ingreso per cápita del hogar).

\section{Desarrollo}

En la tabla 1 comenzamos con la presentación de la relación bivariada entre AF para MCF/D que, a medida que aumenta el ingreso per cápita del hogar y el nivel educativo, así como en los hogares sin NBI, se incrementa la realización de este tipo de actividad física. Además, 
observamos que la Ciudad Autónoma de Buenos Aires es la región donde en mayor medida la población realiza estas prácticas (47.4\%) mientras que el conurbano bonaerense es donde menos se realiza (30.5\%). Por último, vemos que los hombres $(43.0 \%)$ y el grupo etario más joven (49.4\%) son quienes más realizan ejercicio físico; por otr lado, las mujeres (34.4\%) y el grupo de mayor edad (27.3\%) son quienes menos realizan actividades físicas.

Tabla 1. Actividad física para mejorar condición física o hacer deporte en los últimos treinta días según variables seleccionadas. Población residente en zonas urbanas de 5000 habitantes y más de Argentina en el año 2009. Datos en \%.

\begin{tabular}{|c|c|c|c|}
\hline \multicolumn{4}{|c|}{ Actividad física para mejorar la condición física o hacer deporte (últimos treinta días) } \\
\hline & & Sí & No \\
\hline \multirow{5}{*}{ Quintiles de ingreso per cápita del hogar } & 1er quintil & 27.1 & 72.9 \\
\hline & 2do quintil & 33.8 & 66.2 \\
\hline & 3er quintil & 35.0 & 65.0 \\
\hline & 4to quintil & 41.3 & 58.7 \\
\hline & 5to quintil & 53.2 & 46.8 \\
\hline \multirow{4}{*}{ Nivel educativo } & Hasta primario incompleto & 18.8 & 81.2 \\
\hline & Primario completo-secundario incompleto & 31.6 & 68.4 \\
\hline & Secundario completo-superior incompleto & 45.4 & 54.6 \\
\hline & Superior completo & 54.9 & 45.1 \\
\hline \multirow{2}{*}{ NBI } & Hogar con NBI & 25.5 & 74.5 \\
\hline & Hogar sin NBI & 40.5 & 59.5 \\
\hline \multirow{7}{*}{ Región } & $\mathrm{CABA}$ & 47.4 & 52.6 \\
\hline & Conurbano Bonaerense & 30.5 & 69.5 \\
\hline & Pampeana & 41.8 & 58.2 \\
\hline & Noroeste & 37.6 & 62.4 \\
\hline & Noreste & 35.6 & 64.4 \\
\hline & Cuyo & 40.8 & 59.2 \\
\hline & Patagónica & 42.3 & 57.7 \\
\hline \multirow{2}{*}{ Sexo } & Hombre & 43.0 & 57.0 \\
\hline & Mujer & 34.4 & 65.6 \\
\hline \multirow{5}{*}{ Grupo etario } & 18 a 24 años & 49.4 & 50.6 \\
\hline & 25 a 34 años & 42.5 & 57.5 \\
\hline & 35 a 49 años & 37.4 & 62.6 \\
\hline & 50 a 64 años & 34.8 & 65.2 \\
\hline & 65 años y más & 27.3 & 72.7 \\
\hline
\end{tabular}

Fuente: elaboración propia en base a la ENFR 2009. 
En la tabla 2, presentamos una regresión logística para analizar el efecto que las distintas variables independientes tienen sobre la realización de AF para MCF/D una vez controladas el resto de las variables. En un primer modelo incluimos las variables de nivel educativo, ingreso per cápita del hogar, presencia de necesidades básicas insatisfechas (NBI) y la región de residencia. En un segundo modelo incluimos también las variables de sexo y grupo etario. En los siguientes tres modelos incluimos todas las variables del modelo 2 más la interacción de sexo con otra variable: en el modelo 3 incluimos la interacción de sexo con quintil de ingreso per cápita del hogar; en el modelo 4, de sexo con grupo etario; y en el modelo 5, de sexo con nivel educativo.

En el modelo 1, observamos que, a medida que aumenta el quintil de ingreso per cápita del hogar, aumentan las chances de realizar AF para $\mathrm{MCF} / \mathrm{D}$, de forma tal que quienes pertenecen al 5 to quintil tienen casi dos veces (1.95) más chances que los del primer quintil de realizar actividad física. También observamos que la posibilidad de realizar actividad física también aumenta a mayor nivel educativo formal, de forma tal que quienes tienen estudios superiores tienen casi cuatro veces (3.71) más chances de realizar esta AF que quienes tienen estudios primarios incompletos. A su vez, las personas que viven en hogares sin NBI tienen 1.22 veces más chances de realizar AF para MCF/D que las que lo hacen en hogares con NBI.

Por último, es interesante observar lo que sucede en las distintas regiones. A diferencia de lo que observamos en el tabla 1, vemos que la única diferencia significativa entre la cABA y el resto de las regiones es la que existen con el Conurbano Bonaerense. Ello en parte se debe a que la mayor actividad física realizada en la Ciudad de Buenos Aires está relacionada con que a que allí reside la población con mayores niveles educativos y de más dinero. Una vez controladas estas variables, no hay diferencias con el resto de las regiones, con excepción del conurbano (quienes residen allí tienen $43 \%$ menos chances de realizar esta práctica que los de CABA).

Tabla 2. Regresión logística: chances relativos de factores que inciden en la realización de actividad física para mejorar la condición física o hacer deporte en los últimos treinta días. Población residente en zonas urbanas de 5000 habitantes y más de Argentina en el 2009.

Modelo 1 Modelo 2 Modelo 3 Modelo 4 Modelo 5

Quintiles de ingreso per cápita del hogar (1ero referencia)

2do quintil

3er quintil

4to quintil

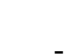

5to quintil

$\begin{array}{ll}1.18^{* *} & 1.23^{* *} \\ 1.15^{* *} & 1.30^{* *} \\ 1.41^{* *} & 1.68^{* *} \\ 1.95^{* *} & 2.34^{* *}\end{array}$

$1.42^{* *}$

$1.22 * *$

$1.23^{* *}$

$1.54^{* *}$

$1.28^{* *}$

$1.29 * *$

$2.15^{* *}$

$1.67^{* *}$

$1.67^{* *}$

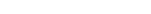
3.19 ** $2.32 *$ $2.33^{* *}$ 
Modelo 1 Modelo 2 Modelo 3 Modelo 4 Modelo 5

Nivel educativo (hasta primario incompleto referencia)

Primario completo-secundario incompleto

Secundario completo-superior incompleto

$1.90 * *$

$3.08 * *$

$3.71^{* *}$

Superior completo

NBI (con NBI referencia)

Región (cABA referencia)

Conurbano Bonaerense

Pampeana

Noroeste

Noreste

Cuyo

Patagónica

Sexo (mujer referencia)

Grupo etario (65 años y más referencia)

18 a 34 años

25 a 34 años

35 a 49 años

50 a 64 años

1er quintil de ingreso per cápita del hogar y mujer referencia

2do quintil y hombre

3er quintil y hombre

4to quintil y hombre

5to quintil y hombre

Rango de edad* Sexo

18 a 24 años y hombre

25 a 34 años y hombre

35 a 49 años y hombre

50 a 65 años y hombre

Hasta primario incompleto y mujer referencia

Primario completo-secundario incompleto

Secundario completo-superior incompleto

Superior completo

Constante

Pseudo R2 de Nagelkerke

Notas: (1) O = No realizó AF; 1 = Realizó AF

${ }^{* *} p<0.01 ;{ }^{*} p<0.05 ;+p>0.05 ;$ N/C: no considerada.

Fuente: elaboración propia en base a la ENFR 2009

$1.22^{* *} \quad 1.36^{* *}$

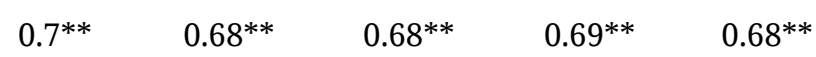

$1.09+1.07+1.07+\quad 1.08+\quad 1.07+$

$1.12+\quad 1.09+\quad 1.08+\quad 1.09+\quad 1.08+$

$1.09+\quad 1.05^{+} \quad 1.04+\quad 1.05^{+} \quad 1.04+$

$1.11+1.10+\quad 1.09+\quad 1.11+\quad 1.09+$

$1.12+1.03+\quad 1.03+\quad 1.04+\quad 1.03+$

$\begin{array}{lllll}\mathrm{N} / \mathrm{C} & 1.46^{* *} & 2.09 * * & 1.19 * & 1.43^{* *}\end{array}$

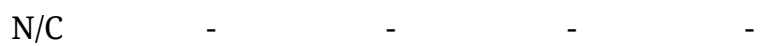

$\mathrm{N} / \mathrm{C} \quad 2.88^{* *} \quad 2.90^{* *} \quad 1.89^{* *} \quad 2.87^{* *}$

$\mathrm{N} / \mathrm{C} \quad 1.88^{* *} \quad 1.90^{* *} \quad 1.44^{* *} \quad 1.87^{* *}$

$\mathrm{N} / \mathrm{C} \quad 1.52^{* *} \quad 1.53^{* *} \quad 1.43^{* *} \quad 1.51^{* *}$

$\mathrm{N} / \mathrm{C} \quad 1.37^{* *} \quad 1.38^{* *} \quad 1.68^{* *} \quad 1.37^{* *}$

$\begin{array}{lllll}\mathrm{N} / \mathrm{C} & \mathrm{N} / \mathrm{C} & - & \mathrm{N} / \mathrm{C} & \mathrm{N} / \mathrm{C}\end{array}$

$\begin{array}{lllll}\mathrm{N} / \mathrm{C} & \mathrm{N} / \mathrm{C} & 0.75^{* *} & \mathrm{~N} / \mathrm{C} & \mathrm{N} / \mathrm{C}\end{array}$

$\begin{array}{lllll}\mathrm{N} / \mathrm{C} & \mathrm{N} / \mathrm{C} & 0.71^{* *} & \mathrm{~N} / \mathrm{C} & \mathrm{N} / \mathrm{C}\end{array}$

$\mathrm{N} / \mathrm{C} \quad \mathrm{N} / \mathrm{C} \quad 0.61^{* *} \quad \mathrm{~N} / \mathrm{C} \quad \mathrm{N} / \mathrm{C}$

$\begin{array}{lllll}\mathrm{N} / \mathrm{C} & \mathrm{N} / \mathrm{C} & 0.54^{* *} & \mathrm{~N} / \mathrm{C} & \mathrm{N} / \mathrm{C}\end{array}$

$\mathrm{N} / \mathrm{C} \quad \mathrm{N} / \mathrm{C} \quad \mathrm{N} / \mathrm{C} \quad$ - $\quad \mathrm{N} / \mathrm{C}$

$\begin{array}{lllll}\mathrm{N} / \mathrm{C} & \mathrm{N} / \mathrm{C} & \mathrm{N} / \mathrm{C} & 2.28^{* *} & \mathrm{~N} / \mathrm{C}\end{array}$

$\mathrm{N} / \mathrm{C} \quad \mathrm{N} / \mathrm{C} \quad \mathrm{N} / \mathrm{C} \quad 1.69 * * \quad \mathrm{~N} / \mathrm{C}$

$\begin{array}{lllll}\mathrm{N} / \mathrm{C} & \mathrm{N} / \mathrm{C} & \mathrm{N} / \mathrm{C} & 1.13+ & \mathrm{N} / \mathrm{C}\end{array}$

$\begin{array}{lllll}\mathrm{N} / \mathrm{C} & \mathrm{N} / \mathrm{C} & \mathrm{N} / \mathrm{C} & 0.64^{* *} & \mathrm{~N} / \mathrm{C}\end{array}$

$\mathrm{N} / \mathrm{C} \quad \mathrm{N} / \mathrm{C} \quad \mathrm{N} / \mathrm{C} \quad \mathrm{N} / \mathrm{C} \quad-$

$\begin{array}{lllll}\mathrm{N} / \mathrm{C} & \mathrm{N} / \mathrm{C} & \mathrm{N} / \mathrm{C} & \mathrm{N} / \mathrm{C} & 1.07+\end{array}$

$\begin{array}{lllll}\mathrm{N} / \mathrm{C} & \mathrm{N} / \mathrm{C} & \mathrm{N} / \mathrm{C} & \mathrm{N} / \mathrm{C} & 1.09+\end{array}$

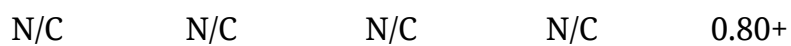

$\begin{array}{lllll}0.17 & 0.09 & 0.08 & 0.10 & 0.10+\end{array}$

$\begin{array}{lllll}0.095 & 0.129 & 0.132 & 0.141 & 0.130+\end{array}$

En el modelo 2, comenzamos observando lo que sucede con el efecto de las variables introducidas en el modelo 1 una vez controlado el sexo y la edad. Allí se observa que aumenta 
la magnitud del efecto del ingreso per cápita y del NBI, y disminuye el efecto del nivel educativo. Ello se debe a que las personas pertenecientes a los hogares con más dinero y sin NBI tienen un promedio de edad mayor que las pertenecientes a hogares con menos dinero y con NBi. En cambio, las personas con niveles de estudios más altos tienen un promedio de edad más bajo. Es decir, parte del efecto del nivel educativo que observamos en el modelo 1 se debe a que los grupos etarios más jóvenes tienen mayores niveles educativos y los más jóvenes realizan más actividad física. En cambio, en el caso del ingreso per cápita del hogar y del NBI, el efecto de estas variables se ve disminuido por la edad en el modelo 1 . En cuanto a la región de residencia no se observan diferencias.

Con relación al efecto del grupo etario, observamos que, a medida que aumenta la edad, disminuyen las chances de realizar este tipo de actividad física. Los jóvenes de entre $18 \mathrm{y}$ 24 años tienen casi tres veces (2.88) más chances de realizar actividad física que los adultos mayores de 66 años y más. Mientras, los hombres tienen 1.46 veces más chances de realizarla que las mujeres. Los resultados de los modelos 1 y 2 nos permiten confirmar la hipótesis 1.

En el modelo 3, introducimos la interacción entre sexo y quintil de ingreso per cápita del hogar, lo que nos permitirá poner a prueba la hipótesis 2: son más importantes las diferencias de género entre los sectores de menos recursos. Como era esperable, en las variables que no son parte de la interacción no se ve modificado su efecto. En cambio, observamos que se modifica la magnitud del efecto de las variables sexo y quintil de ingreso per cápita del hogar, a la vez que las interacciones son significativas. A continuación, analizamos lo que significa cada efecto.

Comencemos por el término de la interacción: lo que nos indica es que, tomando como referencia el primer quintil de ingreso per cápita del hogar, a medida que aumenta el quintil de ingreso, son menores las diferencias de género en la realización AF para MCF/D. Dicho de otra forma, la diferencia a favor de los hombres frente a las mujeres para realizar actividad física dentro del primer quintil de ingresos es significativamente mayor a la misma comparación de probabilidades en cada uno de los quintiles restantes. Es decir, se corrobora nuestra hipótesis de trabajo en torno a que las diferencias de género en las chances relativas de realizar AF para McF/D son significativamente más importantes entre los sectores de menores recursos (primer quintil de ingreso per cápita del hogar) que en el resto.

Por otro lado, vemos que tanto las variables sexo como quintil de ingreso per cápita aumentan la magnitud de su efecto sobre la variable dependiente. Ello se debe a que, luego de incorporar los términos de la interacción, los coeficientes de las dos variables independientes originales reflejan el efecto de esa variable, pero solo en la categoría de referencia de la otra variable independiente (31). En el modelo 3 se observa que los hombres tienen dos veces (2.09) más chances de realizar este tipo de actividad física que las mujeres (en el modelo 2 estas chances eran de 1.46). Ello se debe a que este coeficiente muestra el efecto de ser mujer para la categoría de referencia de la otra variable de la interacción (primer quintil de ingreso per 
cápita del hogar). Es decir, lo que nos señala ahora ese coeficiente es que, en el primer quintil de ingreso, los hombres tienen dos veces más chances que las mujeres de realizar AF para McF/D.

Con relación al ingreso sucede algo similar, ya que estos datos nos indican su efecto en la categoría de referencia de la otra variable de la interacción (ser mujer). Por esa razón también aumenta la magnitud de las chances relativas. Si nos centramos en las diferencias entre el primer y el quinto quintil, observamos que en el modelo 3 las mujeres del quinto quintil tienen 3.19 veces más chances de realizar esta práctica física que las mujeres del primer quintil (en el modelo 2 era 2.34). A su vez, podemos observar una tendencia creciente en los coeficientes leídos a medida que avanzamos entre los quintiles intermedios (2, 3 y 4). Los datos muestran que las diferencias generadas por los ingresos para realizar AF para McF/D son más importantes entre las mujeres que entre los hombres.

Por su parte, en el modelo 4 ponemos a prueba la hipótesis $3^{\mathrm{a}}$, que sostiene que las desigualdades de género aumentan con la edad, y la $3 \mathrm{~b}$, que sostiene la relación inversa. Para ello, incorporamos la interacción de sexo y grupo etario. Los resultados de la interacción en el modelo 4 aportan elementos para confirmar la hipótesis 3b y refutar la hipótesis $3^{\mathrm{a}}$. En la interacción se observa, tomando como referencia al grupo de personas de 66 años y más, que las diferencias de género a favor de los hombres son significativamente más importantes entre el grupo de personas de 18 a 24 años y en el grupo de personas de 25 a 34 años. Es decir, en los dos grupos más jóvenes las diferencias de género son significativamente más importantes que en el grupo de mayor edad. Sin embargo, cuando comparamos estos datos con los relativos al grupo de personas de 35 a 49 años, las diferencias de género dejan de ser significativas, mientras que en el grupo de personas de 50 a 65 años las diferencias de género son significativamente menores que las relativas al grupo de 66 años y más. Como conclusión podríamos señalar que, entre los grupos más jóvenes (18 a 24 años y 25 a 34 años), las diferencias de género son significativamente más importantes que entre los grupos de mayor edad (50 a 65 años y 66 y más años). Sin embargo, entre estos dos últimos grupos de edad, las diferencias de género son más marcadas en el grupo de 66 y más que en el de 50 a 65 años.

Por último, cabe señalar lo que ocurre con las otras variables que son parte de la regresión. Al igual que en el modelo 3, las variables que no son parte de la interacción tienen el mismo efecto que en el modelo 2. En cambio, las magnitudes del efecto del sexo y del grupo etario se modifican. Con relación al sexo, observamos que disminuye el efecto (las mayores chances relativas de los hombres con relación a las mujeres son 1.19 en el modelo 4, frente a 1.46 del modelo 2). Ello se debe a que en el modelo 4 estamos viendo el efecto del sexo solo para el grupo etario de 66 años y más (categoría de referencia de la interacción).

A su vez, observamos que la magnitud del efecto de las dos categorías etarias más jóvenes disminuye notablemente. En el modelo 4, el grupo etario más joven pasa a tener 1.89 veces más chances de realizar actividad física, y el grupo de 25 a 34 años pasa a tener 1.44 veces más chances, mientras que en el modelo 2 esas magnitudes eran de 2.90 y 1.90 respectivamente. Ello se debe a 
que estamos analizando el efecto de la edad solo entre las mujeres. Es decir, coincidentemente con los resultados de la interacción, las mayores chances de realizar actividad física entre los grupos más jóvenes en comparación con los de personas de más edad disminuyen cuando nos centramos en las mujeres y no en la población general (hombres y mujeres, como en el modelo 2). Observamos también que las diferencias en el grupo de personas de 35 a 49 años también disminuyen, pero muy levemente (pasan de 1.53 a 1.43). Recordemos que en la interacción destacamos que, para este grupo, las diferencias de género no son significativamente mayores que en el grupo de 66 y más años. Por último, observamos que en el único grupo que aumentan las diferencias es con respecto al grupo de personas de 50 a 65 años. En el modelo 4 las personas (mujeres) de 50 a 65 años tienen 1.68 veces más chances de realizar esta actividad física que las de 66 años y más; mientras que en el modelo 2 (que considera a hombres y mujeres) esas chances eran 1.38 veces mayores. En ese sentido, estos datos también son consistentes con lo que se observa en el término de la interacción, en la que se toma como referencia al grupo de 66 años y más; observamos, pues, que las diferencias de género son estadísticamente significativas menores en el grupo de 50 a 65 años.

Para finalizar nuestro análisis, cabe señalar que en el modelo 5 también ponemos a prueba nuestra hipótesis 2, pero en esta ocasión analizamos el nivel educativo y no el ingreso. Es por ello por lo que incorporamos una interacción entre el nivel educativo y el sexo, y ese dato no es estadísticamente significativo. En este caso, tomamos como referencia a quienes realizaron hasta primaria incompleta y observamos que las diferencias de género no son significativamente diferentes con las existentes en los restantes niveles educativos.

\section{Conclusiones}

—n este artículo, analizamos datos de la enfr 2009 con el propósito de observar las desigualtipo de actividad física, nos referimos a una práctica voluntaria y recreativa que, además de proteger la salud física, es beneficiosa para la calidad de vida, el bienestar emocional y la sociabilidad (29). Mediante técnicas de análisis bivariado, notamos cómo la práctica deportiva y física está estratificada socialmente en beneficio de los varones, los más jóvenes, los grupos con mayores ingresos y niveles educativos, y aquellos que viven en la caBA. $\mathrm{Al}$ introducir los modelos de regresión logística, notamos el peso de cada variable independiente sobre las chances relativas de realizar AF para McF/D una vez controladas las restantes. Así observamos en el modelo 1, por ejemplo, que quienes tienen mayores niveles educativos tienen casi cuatro veces más chances de realizar actividad física en comparación con aquellos con niveles más bajos de educación formal, y que en el quintil 5 se 
duplican las chances respecto del quintil 1. En el modelo 2, al controlar los datos por sexo y grupo etario, observamos que aumenta la magnitud del efecto del ingreso per cápita y del NBI, y disminuye el efecto del nivel educativo. Ello se debe a que las personas con mejores posiciones económicas tienen un promedio de edad mayor, mientras que las personas con mayor educación formal tienen un promedio de edad más bajo. Es decir, parte del efecto del nivel educativo que observamos en el modelo 1 se debe a que los grupos etarios más jóvenes tienen mayo nivel educativo, y los más jóvenes realizan más actividad física; con respecto del ingreso per cápita del hogar y el NBI, el efecto de estas variables se ve disminuido por la edad en el modelo 1. Así mismo, observamos que la consideración de las distintas regiones no resulta significativa, a excepción de la comparación entre CABA y el Conurbano Bonaerense. Cabe destacar, de todas formas, que esta variable resulta poco esclarecedora debido al nivel de agregación de los datos. En particular, en las regiones más extensas se consideran áreas geográficas muy amplias y de esta forma no se contemplan las diferencias internas, lo que impide captar la variedad y la heterogeneidad que componen las mismas. Esta aclaración vale también para la comparación entre el Conurbano y CABA, aun si son significativos los resultados del modelo, ya que ambas áreas geográficas presentan importantes heterogeneidades en su interior. Las diferencias observadas en el nivel agregado pueden explicarse en parte por la mayor disponibilidad de infraestructura pública y privada para hacer ejercicio físico en CABA, así como por la percepción del espacio público como inseguro en las zonas de menores recursosdel Conurbano de Buenos Aires (3-5). Estos resultados corroboran nuestra hipótesis 1: que distintos factores socioeconómicos, geográficos y demográficos tienen un peso propio una vez controlado el efecto del resto, y está en línea con antecedentes de estudios realizados en Argentina (22-26).

Al introducir las interacciones en los modelos de regresión logística pusimos a prueba la hipótesis 2: que las diferencias de género se intensifican entre los sectores peor posicionados en la estructura social. Comprobamos que las diferencias de ingreso acentúan las de género en las chances relativas de realizar AF para MCF/D en detrimento de las mujeres con menores recursos. Es decir, las diferencias de género para realizar AF aumentan a medida que disminuye el nivel de ingreso. Un dato sorprendente es el relativo a la interacción entre sexo y nivel educativo, que no es estadísticamente significativa. Los argumentos teóricos para realizar esta interacción son similares a los de la interacción de ingreso y sexo, y la hipótesis de trabajo es que, a mayor nivel educativo, menores desigualdades de género. Ello indicaría que son los recursos materiales más que los cognitivos y simbólicos los que tienen una interacción con el género en la estratificación de las chances de realizar AF para McF/D. Si bien no hay antecedentes sobre análisis de este tipo en Argentina, destacamos que. a diferencia de nuestros resultados, un metaanálisis sobre estudios realizados en Europa encuentra que son más frecuentes los trabajos que hallan una relación entre nivel educativo 
y la AF en tiempo libre entre las mujeres, mientras que la relación entre AF en tiempo libre e ingreso fue más frecuente entre los hombres (10).

Con respecto a la interacción entre género y edad (hipótesis 3), observamos que en los grupos más jóvenes (18 a 24 años y 25 a 34 años) las diferencias de género son significativamente más importantes que en los grupos de mayor edad (50 a 65 años, y 66 y más años), lo que abona la hipótesis del peso de la maternidad y las responsabilidades por el cuidado sobre las menores chances de disponer de tiempo personal para el ocio y las actividades recreativas. En este sentido, comprobamos la hipótesis 3b, que postula que las diferencias de género para la realización de AF tienden a disminuir a medida que aumenta la edad, rechazando la contrapuesta hipótesis 3a. Sin embargo, entre los dos grupos de mayor edad, son mayores las diferencias de género en el grupo de 66 y más, que en el de 50 a 65 años. Si bien en Argentina no hay antecedentes de análisis de este tipo, la menor incidencia de diferencias de género entre los grupos de mayor edad coincide con lo encontrado por algunos estudios internacionales (12-19), aunque hay otros trabajos que señalan que entre las personas mayores las diferencias pueden aumentar por la socialización en una cultura masculina del deporte (12).

Los resultados anteriores que incorporan interacciones al interior de las regresiones logísticas muestran la relevancia de considerar un enfoque interseccional para comprender la naturaleza multiplicativa de las desigualdades en la práctica de actividad física que los modelos aditivos no pueden captar $(13,14)$. Sería importante poder profundizar en futuros trabajos en los factores explicativos de la interacción del género con el ingreso y con la edad. Señalamos como un factor importante el hecho de que las mujeres jóvenes que son madres y de bajos ingresos tienen poco tiempo disponible por causa de la doble jornada (productiva y reproductiva) lo que puede impedir la realización de AF (32). Desde el punto de vista de las intervenciones políticas, es imperativo tener en cuenta dicha situación a la hora de diseñar políticas públicas que promuevan la realización de Af para estos sectores. Por ejemplo, los programas diseñados para favorecer que estos grupos realicen AF se pueden complementar con otros que liberen el tiempo de cuidado de los niños (como programas deportivos para madres e hijos, o guarderías para los más pequeños). En esta línea, un estudio realizado en Australia muestra que cuando se provee cuidado para los niños aumenta la actividad física en los hogares unipersonales (33). Otra posibilidad que ha mostrado ser efectiva entre mujeres trabajadoras es la promoción de la realización de actividad física en los lugares de trabajo, aunque una dificultad es que los empleadores acepten destinar tiempo para ello (34).

Cabe señalar como una limitación del trabajo el hecho de que solo analizamos la realización de AF para MCF/D durante los últimos treinta días y no tuvimos en cuenta otras dimensiones de importancia para comprender la problemática aquí analizada, como la frecuencia y el lugar de realización, el tipo de actividad realizada, y el vínculo con otras actividades físicas como la laboral, la doméstica y la generada por traslados cotidianos. Destacamos que hemos analizado varias de las temáticas de forma descriptiva en otro trabajo (28) y que en 
este artículo optamos por realizar un análisis multivariado más complejo, que examina solo una de estas dimensiones.

\section{Contribución de los autores}

os cuatro autores procesaron y analizaron los datos, buscaron bibliografía y redactaron
el artículo.

\section{Agradecimientos}

Ede cuidado de la salud en la periferia de Buenos Aires: desigualdad social, territorio y $^{\text {lartiolo fue realizado en el marco delos Proyectos uвасут 20020170100024BA “Experiencias }}$ cultura”, ріст 2017-1391 "Desigualdad social, cultura y salud en un barrio de la periferia de Buenos Aires” y PRII (FSOC-UBA) “Desigualdades sociales en estilos y modos de vida vinculados con la salud en zonas urbanas de Argentina, 2007-2014”. Además, el artículo fue parcialmente elaborado en el contexto de la Red INCASI, un proyecto europeo que ha recibido fondos del Programa de Investigación e Innovación Horizonte 2020 de la Unión Europea, bajo la Marie Skłodowska-Curie ga No 691004 y es coordinado por el Dr. Pedro López-Roldán. Este trabajo refleja únicamente la opinión de los autores y la agencia no es responsable del uso que pueda hacerse de la información que contiene.

\section{Referencias}

1. Breihl J. La epidemiología crítica: una nueva forma de mirar la salud en el espacio urbano. Salud Colect. 2010;6(1):83-101. Doi: 10.18294/sc.2010.359

2. Menéndez E. De sujetos, saberes y estructuras: introducción al enfoque relacional en el estudio de la salud colectiva. $1^{\text {a }}$ edic. Buenos Aires: Lugar Editorial; 2009.

3. Moore LV, Diez Roux AV, Evenson KR, McGinn P, Brines SJ. Availability of recreational resources in minority and low socioeconomic status areas. Am J Prev Med. 2008;34(1):1622. Doi: 10.1016/j.amepre.2007.09.021

4. Freidin B, Wilner A, Ballesteros M, Krause, M. Salud, riesgo ambiental y territorio: las experiencias de mujeres que viven en un barrio de clase popular en la periferia de Buenos Aires. Buenos Aires: Teseo Press; 2018.

5. Parra DC, McKenzie TL, Ribeiro IC, Ferreira Hino AA, Dreisinger M, Coniglio K, et al. Assessing physical activity in public parks in Brazil using systematic observation. Am. J Public Health Res. 2010;100(8):1420-26. Doi: 10.2105/AJPH.2009.181230 
6. Ross, C. Collective Threat, Trust, and the Sense of Personal Control. J Health Soc Behav. 2011;52(3):287-96. Doi: 10.1177/0022146511404558

7. Diez-Roux A, Mair C. Neighborhoods and health. Ann N Y Acad Sci. 2010;1186:125-45. Doi: 10.1111/j.1749-6632.2009.05333.x

8. Humpel N, Owen N, Leslie E. Environmental factors associated with adults participation in physical activity. Am J Prev Med. 2002; 22(3):188-99.

9. Stahl T, Rütten A, Nutbeam D, Bauman A, Kannas L, Abel T, et al. The importance of the social environment for physically active lifestyle-results from an international study. Soc Sci Med. 2001;52(1):1-10.

10. Beenackers MA, Kamphuis CB, Giskes K, Brug J, Kunst AE, Burdorf A, et al. Socioeconomic inequalities in occupational, leisure-time, and transport related physical activity among European adults: a systematic review. Int J Behav Nutr Phys Act. 2012;9(1):116. Doi: 10.1186/1479-5868-9-116

11. García Ferrando M, Llopis Goig R. Estructura social de la práctica deportiva. En García Ferrando M, Puig Barata N, Lagardera Otero F, Llopis Goig R, Vilanova Soler A, editores. Sociología del deporte; capítulo 2. 4ta edición. Madrid: Alianza Editorial; 2017. p. 39-68.

12. Tischer U, Hartmann-Tews I, Combrink C. Sport participation of the elderly-the role of gender, age, and social class. Eur Rev Aging Phys Act. 2011;8:83-91. Doi: 10.1007/ s11556-011-0087-8

13. Abichahine H, Veenstra G. Inter-categorical intersectionality and leisure-based physical activity in Canada. Health Promot Int. 2017;326:91-701. Doi: 10.1093/heapro/daw009

14. Hormiga-Sánchez CM. Perspectiva de género en el estudio de la práctica de actividad física. Rev Cienc Salud. 2015;13(2):233-48. Doi: 10.12804/revsalud13.02.2015.08

15. Ray R. An Intersectional Analysis to Explaining a Lack of Physical Activity Among Middle Class Black Women. Sociology Compass. 2014 jun;8(6):780-91. Doi: 10.1111/soc4.12172

16. Hargreaves J. Promesa y problemas en el ocio y los deportes femeninos. En Brohm M, Bourdieu P, Dunning E, Hargreaves J, Todd T, Young K, editores. Materiales de sociología del deporte. Madrid: Ediciones La Piqueta; 1993. p. 109-132.

17. Frisby W, Crawford S, Dorer T. Reflections on Participatory Action Research: The Case of Low-Income Women Accessing Local Physical Activity services. Journal of Sport Management. 1997; 11(1):8-28.

18. Boersma MK. Low incomes, fast lives? Exploring everyday temporalities of Filipina domestic workers in Hong Kong. Time \& Society. 2016; 25(1):117-37. Doi: 10.1177/0961463X15609807

19. Martínez del Castillo J, González Rivera MD, Jiménez-Beatty Navarro JE, Graupera Sanz JS, Marín Rodríguez M, Campos Izquierdo A, et al. Los hábitos de actividad física de las mujeres mayores en España. Rev Int Cienc Deporte. 2009;14(5):81-93. Doi: 10.5232/ ricyde2009.01407

20. Mosquera González MJ. ¿Las mujeres no hacen deporte porque no quieren?, ¿Los hombres practican el deporte que quieren?: el género como variable de análisis de la práctica deportiva de las mujeres y de los hombres. En: IV Ciclo de Conferencias Xénero, Actividade 
Física e Deporte; 2012-2013. Coruña: Universidade da Coruña Servizo de Publicacións, [internet] 2014 [citado 2019 feb 05. Disponible: http://hdl.handle.net/21183/13594.

21. Puig Barata N, Mosquera MJ. Edad y deporte. En: García Ferrando M, Puig Barata N, Lagardera Otero F, Llopis Goig R, Vilanova Soler A, editores. Sociología del deporte. 4ta ed. Madrid: Alianza Editorial; 2017. p. 125-43.

22. Gómez Rojas G, Grinszpun M, Seid G, editores. Clases de deporte y deportes de clase. La distribución de los gustos y prácticas deportivas en el espacio social. En: VII Jornadas de Sociología de la UNLP "Argentina en el escenario latinoamericano actual: debates desde las ciencias sociales”; La Plata: UNLP, 2012.

23. Linetzky B, De Maio F, Ferrante D, Konfino J, Boissonnet C. Sex-stratified socio-economic gradients in physical inactivity, obesity, and diabetes: evidence of short-term changes in Argentina. Int J Public Health. 2013;58(2):277-84.

24. Riveiro M, Gómez V, editores. Profundizando el análisis del uso diferencial del tiempo libre, desde el género y la clase social. En: VII Jornadas de Sociología de la UNLP “Argentina en el escenario latinoamericano actual: debates desde las ciencias sociales”; La Plata, Argentina. La Plata: UNLP, 2012.

25. Observatorio de la Deuda Social Argentina. Desarrollo humano e integración social en la argentina urbana 2010-2016. Documento estadístico serie del bicentenario (2010-2016). Buenos Aires: UCA; 2017.

26. Tarducci G, Barengo N, Morea G, Gárgano S, Gandini A, Paganini A, et al. Relación entre el nivel de escolaridad y el patrón de actividad física en Balcarce, Argentina. Hacia promoc Salud. 2016;21(2):89-98.

27. Ministerio de Salud. Segunda Encuesta Nacional de Factores de Riesgo. Buenos Aires: Ministerio de Salud; 2011.

28. Ballesteros, M.S., Freidin, B. Desigualdades sociales en la realización de distintas modalidades de actividad física en Argentina. Edu Fís y Cien. 2019;21(1):1-14. Doi: $10.24215 / 23142561 \mathrm{e} 067$

29. WHO. Global action plan on physical activity 2018-2030: more active people for a healthier world. Geneva: WHO; 2018.

30. Ortiz Hernández L. Disparidad en la disponibilidad de infraestructura para la actividad física deportiva en los municipios de México. Rev Salud Publica Nutr. 2005;6(4).

31. Jaccard J. Interaction effects in logistic regression. En: Sage University Papers Series on Quantitative Applications in the Social Sciences. Thousand Oaks, CA: Sage Publications; 2001.

32. Freidin B, Krause M, Balllesteros MS, Wilner, A. Physical activity among adult women living in a poor neighborhood of Metropolitan Buenos Aires: Material constraints, family responsibilities, and territorial resources. En: Congreso Latin American Studies Association (LASA); Boston: LASA; 2019.

33. Azar D, Naughton GA, Joseph GW. Physical Activity and Social Connectedness in SingleParent Families. Leisure Studies. 2009;28(3):349-358. Doi: 10.1080/02614360903046656

34. García LV, Ramos DM, Páez DC, Pedroza LM, Mendoza D. Impacto de un programa de promoción de actividad física en mujeres trabajadoras de dos empresas de Bogotá. Rev Cienc Salud. 2011;9(3):237-49. 